\section{A Really COOL Image Database Environment:}

Jim Hilton, Advanced Data Base Systems

Growing your own microscopy client/server image database requires addressing many issues. If you are going to grow your own, the following ideas will get you "out of the box."

The ideal microscopy image database environment might appear like this: The core of the image database would be a very powerful, universally available, extensible, cost effective, flexible and serviceable database engine. The core engine would allow the user to perform fast and seamless SQL searches across a large database without reducing the available network bandwidth. It would be able to take advantage of current and future technologies (i.e., 64 bit processing, $1 \mathrm{~GB}$ networks, digital, video, multi media, etc.) The same core would be able to share information with other databases, applications and platforms. What an odd concept.

According to Bill Gates, the best operating system for the ideal database is naturally MS Windows NT. The author and most large companies tend to agree with him since NT is very stable, widely held, growing in popularity and easy to administer. NT coupled with MS Access as a microscopy database front-end and MS-SQL 6.5 or Oracle 7.1 as a database server renders a very powerful system. A few advantages of NT include it's ability to handle separate tasks or threads efficiently under heavy load and without blocking users; C2 security; enhanced file stabilization; scaleability and affordability.

Image types from various instruments should have a common port into our ideal database. Image sources would include: video cameras, digital cameras, Twain compliant devices, TV rate and slow scan SEM images, EDX spectra, IR scopes, X-ray diffraction, X-rays, sonic imaging and whatever else comes along. For most systems, images should be

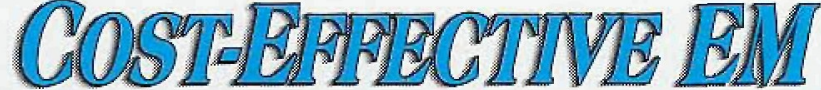 MAGNIETC STUIMDING}

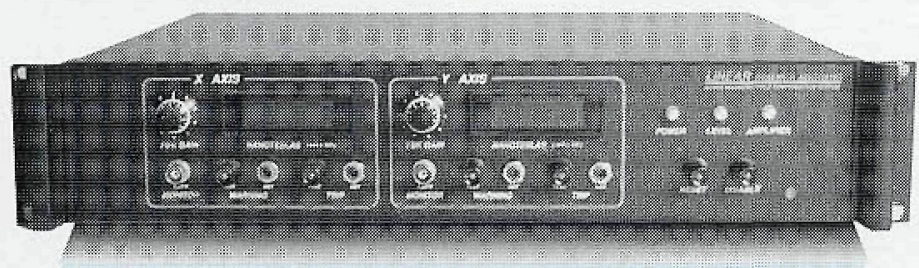

EM sites often require supplemental magnetic shielding to achieve full resolution. LINEAR RESEARCH ASSOCIATES' wideband EMFC$A C$ electronic active-shielding systems dramatically reduce magnetic fields radiating from nearby a.c. power wiring, ground loops, transformers and related sources. EMFC-QDC extended-range models additionally suppress low-frequency magnetic disturbances caused by trains, subways, elevators and equipment such as MRI scanners.

EMFC active-shielding is highly recommended for cost-effective protection of sensitive FEG and PEELS installations.

Linear Research Associates' EMFC equipment is the highestperformance commercially available magnetic field compensation apparatus in the world. EMFC systems feature state-of-the-art engineeringtt and are U.S.-manufactured to exacting standards. Superior performance and reliability are guaranteed!

Call or fax LRA for complete EMFC-series information. We will also gladly assist with any questions you may have regarding site survey, engineering or EMF issues.

\section{LINEAR RESEARCH ASSOCIATES}

5244 Perry City Road - Trumansburg, NY 14886

Phone (607) 387-3411 - Fax (607) 387-7806

t† U.S. Patents $5,465,012 ; 5,469,058$ compressed via JPEG to increase disk space and network bandwidth (compression is a topic for another article.) A mechanism should be inherent to allow storage of "raw" image data when required. Vector (3D, CAD/CAM) and multimedia (AVI, Quick-time, MPEG) files should have a place here as well.

A key factor to any solid image database is "storage". Images, especially from digital cameras and scanners can occupy a lot of disk real-estate. Digital cameras, although not yet widely used are preferred, since their resolution is much better than a video camera. Give some thought to how many and what size images will be taken. How fast does the information need to be written to and read from a disk? How will the information be shared in a client/server environment? How long do you need to keep information? These issues will determine the storage mechanism to be used. A rule of thumb the author uses is: If the information is in the working file use a fast device. When data is near off-line use an expandable system. When the information is to be archived use permanent media.

Our ideal environment should allow viewing of images and data across popular platforms (i.e. UNIX, Macintosh, OS2). Currently there are several emerging technologies for moving and locating information between applications and operating systems. ODBC (open database connectivity) allows various databases to connect over networks. OLE (object linking and embedding) is a mechanism used by Microsoft to link or embed images or data to other applications. Intranet and Internet, the former located inside a corporation or university and the latter being on the World Wide Web, offer features via HTML, Java and Active- $X$, which will affect our entire model in the near feature. An application called "WebHunter" by ADS, Inc. lets the user query an ODBC database from any web browser and view the results with hyperlinked images. "Packet Exchange" lets you compress almost any type of data produced on a Mac, UNIX or Windows computer. The compressed data can then be sent in any manner to any computer that has the Packet Exchange Viewer (free). The viewed data is always in the same form and of excellent quality. OpenDoc for Macintosh and Sun stations offers possibilities for information interchange, but a practical demonstration of this method has not yet been seen.

The creation of a nice looking, well organized reports combining images and data is for the most part not a fun experience. The ideal microscopy image database should possess tools that allow for a wide range of report generation capabilities and a macro language should be available for custom event and report coding. Support for various types of image formats should be inherent. Windows ' 95 and NT both support OLE2 with drag and drop of images into other applications. OLE2 requires support by any Windows image database. Documents generated by the image database should print to any compliant digital printer in the same format as seen on the screen utilizing the maximum resolution of the printer

In a short article it's not practical to cover in depth all aspects of a solid client/server image database solution. However, the issues discussed are extremely relevant and if given consideration will save many hours of mindless work, increase productivity, save on film and disposable budgets, and decrease the cycle time in solving critical problems. Besides the real fun in properly developing an image database, it is very cool

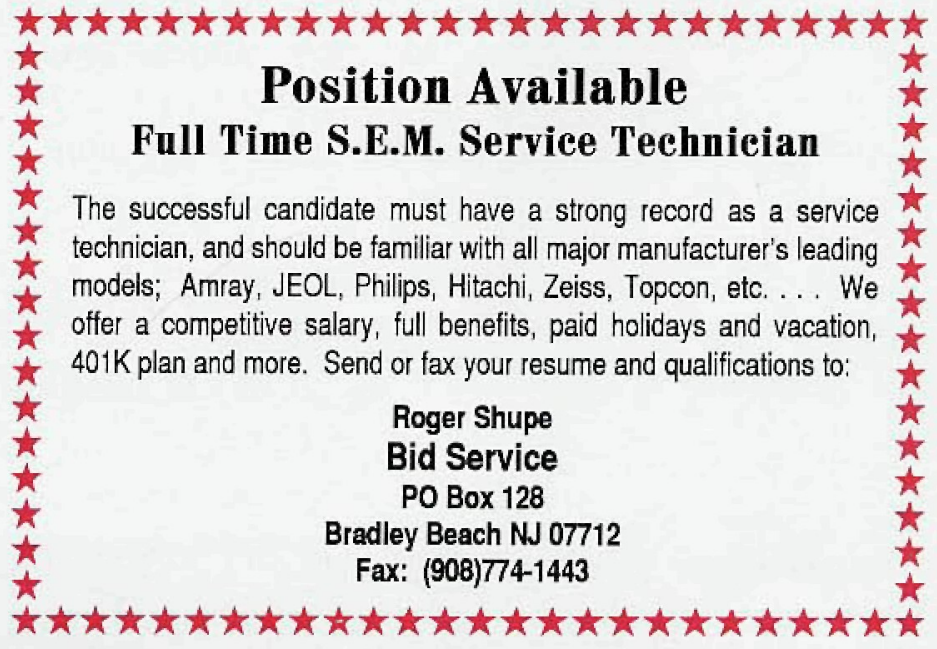




\section{COMMITTED TO EXCELILNCE}

Hydrophilic diamond surface draws water to the edge for easy sectioning.

Flawless, jewel quality diamond crystal, in sizes from 1 to $10 \mathrm{~mm}$.

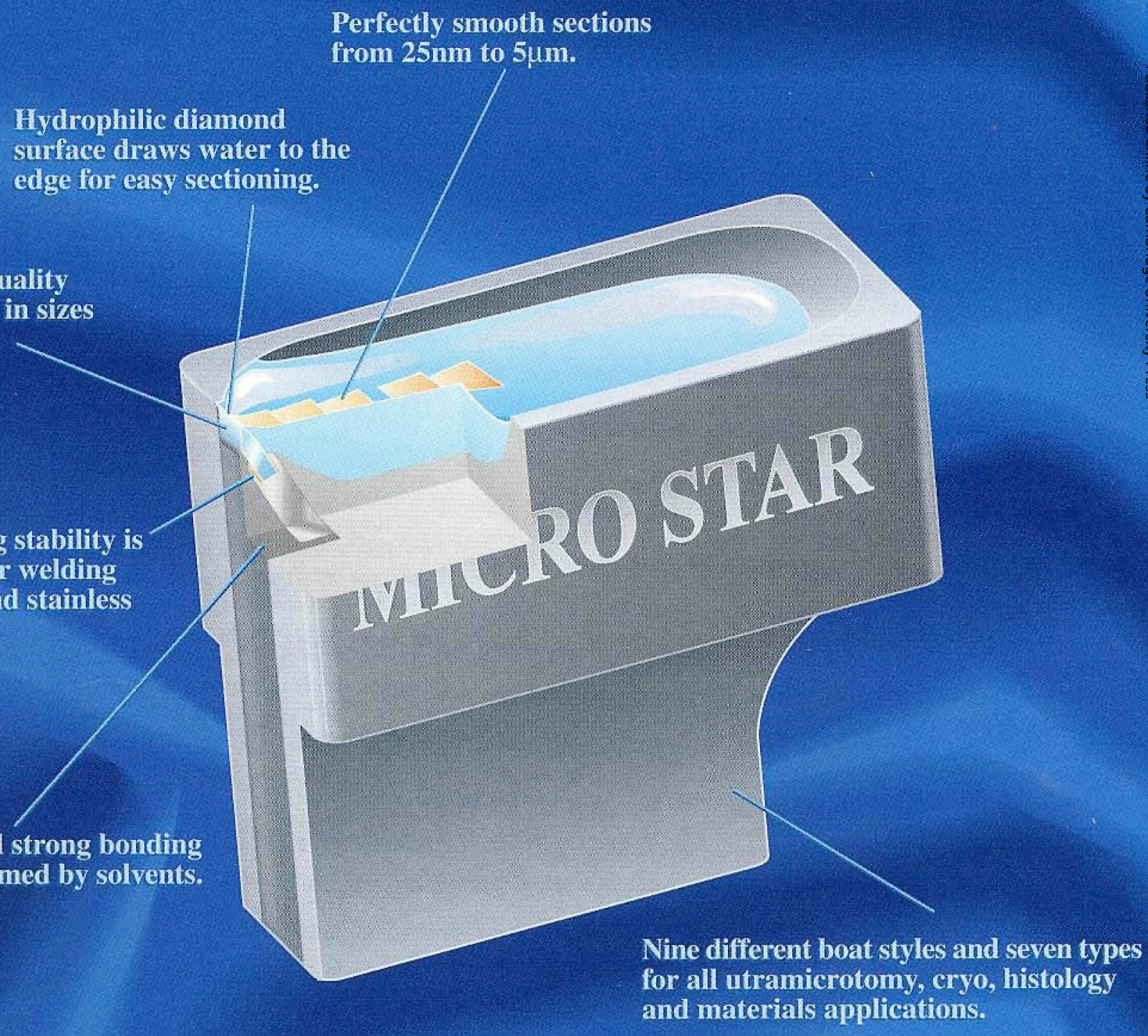

Maximum sectioning stability is assured by molecular welding between diamond and stainless steel shank.

Highly innert and strong bonding material is unharmed by solvents.

Perfectly smooth sections from $25 \mathrm{~mm}$ to $5 \mu \mathrm{m}$.
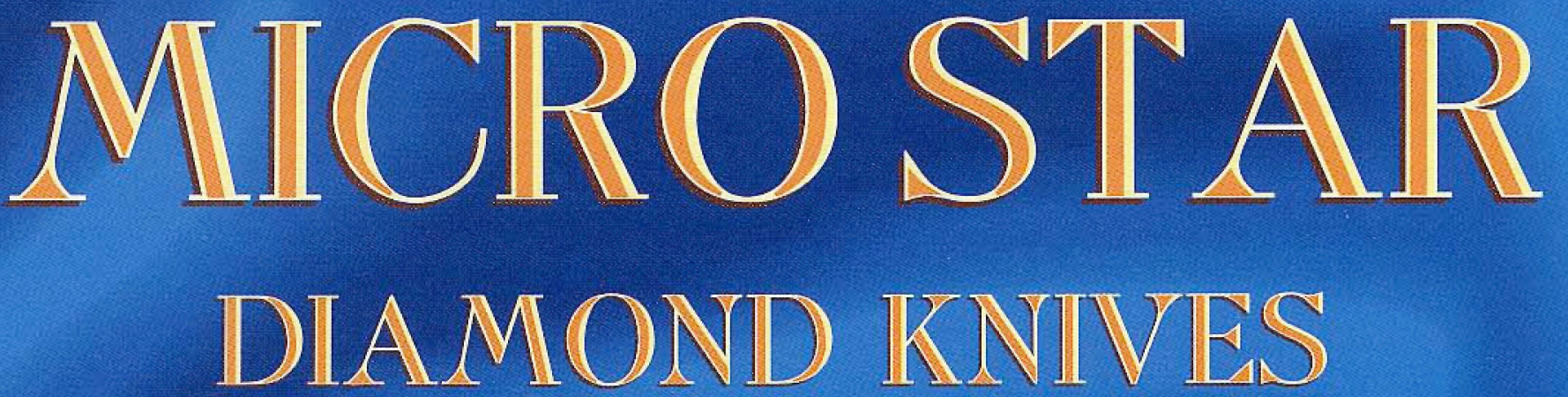

Tel: 8005332509 Tax: 4092949861

E-mail: mistar@msn.com

For full prices and information visit our web site: http://wwwamicrostartech.com/ 